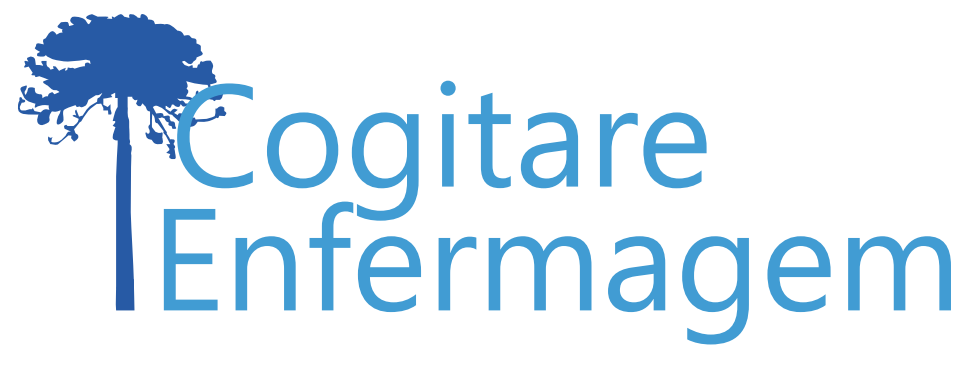

\title{
CONDIÇÕES DE TRABALHO E O IMPACTO NA SAÚDE DOS PROFISSIONAIS DE ENFERMAGEM FRENTE A COVID-19
}

Fernanda Moura D’Almeida Miranda', Leni de Lima Santana², Aline Cecília Pizzolato³, Leila Maria Mansano Saquis ${ }^{4}$

\section{RESUMO}

Objetivo: refletir sobre as condições de trabalho dos profissionais de enfermagem no enfrentamento ao novo coronavírus e apontar o impacto na vida desses profissionais em meio à pandemia.

Desenvolvimento: as fragilidades encontradas no cotidiano laboral dos profissionais de enfermagem são descritas pela literatura nacional e internacional, nas quais estão incluídas as más condições de trabalho, sobrecarga física e mental, baixa remuneração e ausência de Equipamentos de Proteção Individual adequados para o enfrentamento desse agravo.

Considerações finais: esta reflexão pode contribuir para repensar a saúde e segurança dos profissionais de enfermagem visando uma assistência com qualidade e segurança aos pacientes frente a esta doença.

DESCRITORES: Coronavírus; Enfermagem; Saúde do Trabalhador; Impacto Psicossocial; Condições de Trabalho; Riscos Ocupacionais.

\section{COMO REFERENCIAR ESTE ARTIGO:}

Miranda FMA, Santana L de L, Pizzolato AC, Saquis LMM. Condições de trabalho e o impacto na saúde dos profissionais de enfermagem frente a Covid-19. Cogitare enferm. [Internet]. 2020 [acesso em "colocar data de acesso, dia, mês abreviado e ano"]; 25. Disponível em: http://dx.doi.org/10.5380/ce.v25i0.72702.

\section{(c) (1)}

Este obra está licenciado com uma Licença Creative Commons Atribuição 4.0 Internacional.

${ }^{1}$ Enfermeira. Doutora em Enfermagem. Docente de Enfermagem da Universidade Federal do Paraná. Curitiba, PR, Brasil. (C) ${ }^{2}$ Enfermeira. Doutora em Enfermagem. Docente de Enfermagem da Instituto Federal do Paraná. Curitiba, PR, Brasil. 9

${ }^{3}$ Enfermeira. Doutora em Enfermagem. Enfermeira assistencial do Serviço de Atendimento Móvel de Urgência, Secretaria Municipal de Saúde de Curitiba. Curitiba, PR, Brasil. 9

${ }^{4}$ Enfermeira. Doutora em Enfermagem. Docente de Enfermagem da Universidade Federal do Paraná. Curitiba, PR, Brasil. 


\title{
WORKING CONDITIONS AND THE IMPACT ON THE HEALTH OF THE NURSING PROFESSIONALS IN THE CONTEXT OF COVID-19
}

\author{
ABSTRACT \\ Objective: To reflect on the working conditions of the nursing professionals when facing the \\ new coronavirus and to point out the impact on the lives of these professionals in the midst \\ of the pandemic. \\ Development: The weaknesses found in the daily work of the nursing professionals are \\ described by the national and international literature, which include poor working conditions, \\ physical, and mental overload, low pay, and lack of adequate Personal Protective Equipment \\ to cope with this condition. \\ Final considerations: This reflection can contribute to rethink the health and safety of the \\ nursing professionals with a view to providing quality and safe care to the patients in the \\ context of this disease.
}

DESCRIPTORS: Coronavirus; Nursing; Worker's health; Psychosocial impact; Working conditions; Occupational risks.

\section{CONDICIONES DE TRABAJO Y EL EFECTO SOBRE LA SALUD DE LOS PROFESIONALES DE ENFERMERÍA EN ELCONTEXTO DEL COVID-19}

\section{RESUMEN:}

Objetivo: reflexionar sobre las condiciones de trabajo de los profesionales de enfermería en la lucha contra el nuevo coronavirus y señalar el efecto sobre la vida de estos profesionales en medio de la pandemia.

Desarrollo: tanto en la literatura nacional como en la internacional se describen los déficits presentes en la rutina laboral de los profesionales de enfermería, entre los cuales se incluyen las deficientes condiciones de trabajo, la sobrecarga física y mental, la escasa remuneración y la ausencia de Equipos de Protección Personal adecuados para hacer frente a este problema sanitario.

Consideraciones finales: esta reflexión puede ayudar a repensar la salud y la seguridad de los profesionales de enfermería con el propósito de ofrecer asistencia de calidad y seguridad a los pacientes frente a esta enfermedad.

DESCRIPTORES: Coronavirus; Enfermería; Salud del trabajador; Impacto psicosocial; Condiciones de trabajo; Riesgos ocupacionales. 
A pandemia do Severe Acute Respiratory Syndrome Coronavirus 2 (SARS-Cov2), mais conhecida como novo coronavírus 2019 (COVID-19), surge como um desafio para o sistema mundial de saúde, devido ao número de infectados e à demanda de recursos necessários para o seu enfrentamento. Diversos países apresentam números expressivos de pessoas adoecidas demandando internamento e cuidados intensivos em hospitais ${ }^{(1)}$.

O enfrentamento da COVID-19, dentro das instituições de saúde, requer uma diversidade profissional que inclui trabalhadores da saúde e serviços de apoio: serventes, copeiras, seguranças, entre outros. São categorias profissionais com vínculos empregatícios, carga horária e jornadas de trabalho diferenciadas ${ }^{(2)}$. Entre os profissionais de saúde, os Profissionais de Enfermagem (PE), protagonistas do presente estudo, representam aproximadamente 2,2 milhões no Brasil, que atuam em diferentes regiões e em proporções não equalitárias ${ }^{(3)}$. São profissionais que estão na linha de frente no cuidado prestado, independentemente do tipo de atendimento e da situação de saúde, pandêmica ou não.

A essência da profissão dos PE é o processo de cuidar. Esse processo não se restringe ao desenvolvimento de atividades técnicas; envolve também conhecimento científico, sentimentos e emoções. Em uma situação de pandemia, o desgaste físico e mental é comum entre estes trabalhadores. Torna-se conflitante o agir com ética e responsabilidade em meio à sobrecarga de trabalho. As constantes situações de morte e estresse vivenciados em ambientes, muitas vezes, sobrecarregados de pacientes com alto poder de transmissibilidade viral, requerem um atendimento de enfermagem preciso e cauteloso, tanto nos procedimentos técnicos quanto na paramentação e desparamentação rigorosa, conforme recomendado cientificamente ${ }^{(4)}$.

Neste contexto, a Enfermagem se configura como o cerne dos sistemas de saúde em todo o mundo(4). Contudo, as jornadas extensas e condições de trabalho diferenciadas, em razão de diversidades regionais e contratuais, expõem estes profissionais ao risco de adoecimento físico e mental, podendo afastá-los das suas atividades laborais ${ }^{(2,3)}$.

Diante desse cenário, muitos sentimentos florescem, como medo, angústia, preocupação, raiva, sentimento de impotência, entre outros. Tais sentimentos são gerados tanto pela incerteza do que está por vir, como pelo isolamento social imposto aos familiares ${ }^{(5)}$, que em meio à situação de risco, vivenciam o conflito de manterem-se afastados das funções diárias, embora muitas vezes as condições familiares, financeiras e sociais não os permitam essa opção.

Para manter a segurança de seus familiares, os PE necessitam realizar procedimentos diferenciados de higienização em suas residências ${ }^{(6)}$, fabricar ou comprar seus próprios Equipamentos de Proteção Individuais (EPI), devido ao receio da falta deles nos ambientes de trabalho. Diante destes desafios, o objetivo desse estudo é refletir sobre as condições de trabalho no enfrentamento ao novo coronavírus e o impacto na saúde dos PE.

\section{DESENVOLVIMENTO}

\section{As condições de trabalho dos profissionais de enfermagem no enfrentamento a COVID-19}

Geralmente, as condições de trabalho dos PE incluem extensas jornadas, ritmo intenso, desvalorização profissional, conflitos interpessoais, entre outros fatores desencadeantes de desgastes físicos e psíquicos ${ }^{(2)}$. No momento de pandemia, estas condições são potencializadas pelo número de pessoas infectadas e pela escassez de EPIs adequados, situações que elevam os desgastes devido ao medo de infectar-se ou de transmitir o 
vírus aos entes queridos ${ }^{(7)}$. $\bigcirc$ trabalho para as equipes de saúde, subitamente, tornou-se assustador pela insegurança pessoal|(8).

Os PE vivenciam um dilema ético e moral: ao assistir os pacientes sem a utilização dos EPI adequados, colocam em risco a sua vida, a dos pacientes, da equipe de saúde e dos entes queridos. Entretanto, ao negar atendimento aos pacientes em estado de urgência/ emergência, podem ser responsabilizados criminalmente, conforme Art.135 do Código Penal Brasileiro( ${ }^{(9)}$, ainda que amparados pelos conselhos de classe.

De acordo com o Código de Ética dos profissionais de enfermagem, em seu Art. 13, é direito dos PE:

[...] suspender as atividades, individuais ou coletivas, quando o local de trabalho não oferecer condições seguras para o exercício profissional e/ou desrespeitar a legislação vigente, ressalvadas as situações de urgência e emergência, devendo formalizar imediatamente sua decisão por escrito e/ou por meio de correio eletrônico à instituição e ao Conselho Regional de Enfermagem ${ }^{(10: 26)}$.

O Art. 76, em que são abordadas as proibições, assegura ao PE a possibilidade de "negar assistência de enfermagem em situações de urgência, emergência, epidemia, desastre e catástrofe ${ }^{(10: 32) "}$ se o exercício da função oferecer risco à sua integridade física(10). Esses artigos asseguram aos PE o direto de negar atendimento aos pacientes com a COVID-19 sem os EPls adequados ou em condições inseguras de trabalho. Entretanto, esse é o dilema ético e moral enfrentado no cotidiano do trabalho por esses profissionais, que arriscam suas vidas em prol de seus pacientes.

Neste cenário, o valor da vida dos PE e sua responsabilidade civil entram em conflito, podendo trazer consequências para sua saúde e segurança no desempenho de suas atividades laborais. Faz-se necessário e urgente que os PE tenham EPIs adequados e sejam capacitados em serviço para o seu uso correto ${ }^{(11)}$.

Da mesma forma, é necessário ter cautela na definição de ações emergenciais, de forma que o trabalhador de saúde não seja penalizado e ainda mais exposto ao risco. A exemplo da publicação da Medida Provisória (MP) n 927/2020, que flexibiliza as leis trabalhistas para o enfrentamento dessa emergência na Saúde Pública. Entre as disposições desta MP, está a permissão da prorrogação da jornada de trabalho dos profissionais de saúde contratados em regime celetista. O Art. 26 permite ao empregador prorrogar a jornada de trabalho; adotar escalas de horas suplementares que variam da $13^{a}$ até a $24^{a}$ hora do intervalo inter jornada, e prevê a compensação da carga horária suplementar por meio de banco de horas ou hora extra, com um prazo de até 18 meses para que seja realizada(12).

É prudente repensar as escalas de trabalho dos PE de modo a diminuir o desgaste físico e emocional. É preciso considerar que a paramentação rigorosa e adequada, bem como a necessidade de economizar EPls, dificulta a realização de funções fisiológicas como alimentar-se, hidratar-se ou ir ao banheiro, devido ao tempo dispendido para a paramentação e desparamentação entre os procedimentos ${ }^{(12)}$.

A literatura reforça que o aumento da conscientização sobre a proteção pessoal, fornecimento de EPI adequado, em número suficiente, com treinamento de acordo com protocolos nacionais e internacionais, podem contribuir para a redução do risco de infecção em profissionais de saúde ${ }^{(13)}$. Apesar de treinamento intenso e de procedimentos técnicos corretos, ainda existe o risco da exposição biológica durante a atividade profissional, muitas vezes culminando na contaminação do trabalhador. Devido à característica das atividades técnicas, a sobrecarga e o cansaço, tal exposição pode ocorrer e causar afastamento laboral|(11) temporário ou até evoluir para óbito do profissional.

\section{O impacto na saúde do trabalhador de enfermagem}


Estudos ${ }^{(14-16)}$ demonstram que os profissionais de saúde, entre eles os PE, estão sendo acometidos pela COVID-19. Na Espanha, 13\% dos casos foram confirmados em profissionais da saúde, com relato de óbitos entre enfermeiros ${ }^{(14)}$. Na Itália, cerca de $20 \%$ dos profissionais de saúde foram infectados, com 100 óbitos entre médicos e 26 entre enfermeiros $^{(15)}$. Na China, foram 3.000 profissionais infectados com 22 mortes ${ }^{(16)}$, e no Irã e na Indonésia também há relatos de óbitos entre enfermeiros ${ }^{(17)}$.

No Brasil, ainda não há dados oficiais sobre o número de profissionais de saúde acometidos pela COVID-19. Como iniciativa do Conselho Federal de Enfermagem, foi desenvolvido um observatório no qual os PE podem relatar seu adoecimento. Até o dia 13 de abril de 2020, foram registrados 1.203 casos de adoecimento e 18 óbitos ${ }^{(18)}$. Acreditase que esse número seja subestimado, por ser uma notificação voluntária. Esses dados demonstram a necessidade de ações protetivas que permitam o cuidado integral à saúde do PE, com a implementação de protocolos que possam reduzir os riscos de contaminação durante a atividade laboral.

Recomenda-se a padronização dos EPIs preconizados por órgãos nacionais e internacionais como o Centers for Disease Control and Prevention, Occupational Safety and Health Administration, National Institute for Occupational Safety and Health, Organização Mundial de Saúde (OMS), e o Ministério da Saúde.

As orientações sobre o uso de EPI podem ter diversas interpretações e divergir na indicação, como em relação ao uso de macacão ou capote impermeável para a assistência ao paciente infectado. Estas ações se justificam pela diversidade de informações e pelo distanciamento de protocolos, guidelines e experiências internacionais ${ }^{(16)}$. Assim, o apoio dos gestores no enfrentamento dos desafios deve ser diário. O repasse de orientações sobre as medidas de prevenção é essencial para o gerenciamento do estresse causado pelas atividades laborais ${ }^{(11)}$, e pelo excesso de informação das mídias.

Outra problemática decorrente da falta de EPI refere-se à reutilização da máscara N95. Em Nota Técnica publicada pela Agência Nacional de Vigilância Sanitária(19) em 21 de março de 2020, recomendou-se que, em caráter excepcional, os profissionais de saúde poderiam utilizá-la, caso estivessem em boas condições, por várias vezes durante um mesmo plantão de até 12 horas. Entretanto, essa informação foi suprimida na publicação de 31 de março de 2020, permitindo o reuso por um período maior ou um número de vezes maior que o indicado pelo fabricante ${ }^{(20)}$. Evidencia-se que os protocolos são criados, muitas vezes, para atender às necessidades dos serviços, por vezes sem evidências científicas ou respaldo por organizações internacionais, colocando a saúde do trabalhador em risco.

Embora medidas preventivas sejam tomadas, os profissionais de saúde vivenciam situações sem precedentes, tendo que tomar decisões difíceis que podem ocasionar agravos psicológicos a longo prazo, causados por danos morais(21). Assim, é vital que os governantes e gestores reconheçam o valor desses profissionais e os tratem com humanidade, garantindo sua segurança e saúde ${ }^{(22)}$.

Em uma tentativa de minimizar as consequências deste agravo na saúde física e mental dos profissionais, observa-se nas mídias sociais e em canais oficiais a solidariedade de especialistas em saúde mental na oferta de apoio emocional e orientações sobre hábitos saudáveis, visando à manutenção da saúde desses profissionais.

Mesmo em meio às adversidades, a Enfermagem demostra o seu protagonismo e, no ano dedicado a profissão pela World Health Assembly com a chamada para "Nurses and Midwives clean care is in your hands"(23) e por meio da campanha "Nursing Now"(24), cujo um dos lemas é: "onde há vida há enfermagem", em meio aos desafios e ao custo de muitos sacrifícios, vislumbra um reconhecimento social acerca da sua importância nos sistemas de saúde mundiais.

No enfrentamento desta pandemia, destaca-se o papel da Enfermagem nas ações de vigilância, prevenção, controle da transmissão do vírus, assistência aos enfermos, pesquisas sobre a COVID-19 e nas orientações à comunidade. Reforça-se o olhar atento da 
profissão ao cuidado do ser humano, do ambiente, da família e coletividade, com empatia e acolhimento.

\section{CONSIDERAÇÕES FINAIS}

Entende-se que a Enfermagem vivencia um momento ímpar decorrente da pandemia da COVID-19, pela sobrecarga de trabalho, pela especificidade da alta transmissão do vírus e pela manipulação de equipamentos específicos de proteção. Uma experiência vivenciada tanto pela rede pública quanto privada no país, e até mesmo em âmbito mundial.

Acredita-se na Enfermagem como um elo na corrente multiprofissional em saúde no enfrentamento a COVID-19, com foco na vida humana, atentando para a saúde do trabalhador e a segurança do paciente. Considerado um momento de se reinventar e reaprender diante de um cenário desafiador ao trabalhador e às instituições, a presença e posicionamento dos conselhos e associações de classe são indispensáveis.

Salienta-se que este artigo não pretende dar finitude ao tema, mas fomentar reflexões de como a Enfermagem vem enfrentando a pandemia da COVID-19.

\section{REFERÊNCIAS}

1. World Health Organization (WHO). Health workers exposure risk assessment and management in the context of COVID-19 virus. [Internet]. Geneva: WHO; 2020 [acesso em 06 abr 2020]. Disponível em: https://apps.who.int/iris/bitstream/handle/10665/331340/WHO-2019-nCov-HCW risk assessment2020.1-eng.pdf?sequence=1\&isAllowed=y.

2. Santana $L$ de $L$. Riscos psicossociais e saúde mental em ambiente hospitalar: com a voz o trabalhador [tese]. Curitiba (PR): Universidade Federal do Paraná; 2018.

3. Conselho Federal de Enfermagem. Saúde de Profissionais de Enfermagem é foco em tempos de Covid-19 [Internet]. Brasília: COFEN; 2020. [acesso em 06 abr 2020]. Disponível em: http://www.cofen. gov.br/saude-de-profissionais-de-enfermagem-e-foco-em-tempos-de-covid-19 78321.html.

4. Jackson D, Bradburry-Jones C, Baptiste D, Gelling L, Morin K, Neville S, et al. Life in the pandemic: some reflections on nursing in the contexto of COVID-19. J Clin Nurs. [Internet]. 2020 [acesso em 15 abr 2020]; Disponível em: http://doi.org/10.1111/jocn.15257.

5. Ministério da Saúde (BR). Saúde regulamenta condições de isolamento e quarentena [Internet]. Brasília: Ministério da Saúde; 2020 [acesso em 06 abr 2020]. Disponível em: https://www.saude.gov.br/noticias/ agencia-saude/46536-saude-regulamenta-condicoes-de-isolamento-e-quarentena.

6. Ministério da Saúde (BR). Secretaria de Atenção Especializada à Saúde. Departamento de Atenção Hospitalar, Domiciliar e de Urgência. Protocolo de manejo clínico para o novo coronavírus [Internet]. Brasília: Ministério da Saúde; 2020 [acesso em 06 abr 2020]. Disponível em: https://portalarquivos2. saude.gov.br/images/pdf/2020/fevereiro/05/Protocolo-de-manejo-clinico-para-o-novo-coronavirus-2019ncov.pdf.

7. Editorial. COVID-19: protecting health-care workers. The Lancet [Internet]. 2020 [acesso em $06 \mathrm{abr}$ 2020]; 395. Disponível em: https://doi.org/10.1016/S0140-6736(20)30644-9.

8. Fiona G. Protect our healthcare workers. BMJ [Internet]. 2020 [acesso em 06 abr 2020]; 369. Disponível em: https://doi.org/10.1136/bmj.m1324.

9. Brasil. Decreto-Lei n. 2.848, de 07 de dezembro de 1940. Código Penal. Diário Oficial da União [Internet]. Rio de Janeiro, $31 \mathrm{dez}$ 1940. [acesso em 30 abr 2020]. Disponível em: http://www.planalto.gov. 
10. Conselho Federal de Enfermagem. Legislação dos Profissionais de Enfermagem [Internet]. Brasília: COFEN, 2020. [acesso em 06 abr 2020]. Disponível em: http://biblioteca.cofen.gov.br/wp-content/ uploads/2019/11/C\%C3\%B3digo-de-\%C3\%89tica-dos-profissionais-de-Enfermagem.pdf.

11. Huang L, Lin G, Tang L, Yu L, Zhou Z. Special attention to nurses' protection during the COVID-19 epidemic. Critical Care [Internet]. 2020 [acesso em 06 abr 2020]; 24(120). Disponível em: https://ccforum. biomedcentral.com/articles/10.1186/s13054-020-2841-7.

12. Brasil. Medida Provisória n. 927, de 22 de março de 2020. Dispõe sobre as medidas trabalhistas para o enfrentamento do estado de calamidade públicas reconhecido pelo Decreto Legislativo $n^{\circ} 6$, de 20 de março de 2020, e da emergência de saúde pública de importância internacional decorrente do coronavírus (COVID-19), e dá outras providências. Diário Oficial da União. [Internet] 22 mar 2020. [acesso em 30 abr 2020]. Disponível em: http://www.in.gov.br/en/web/dou/-/medida-provisoria-n-927-de-22-de-marco-de-2020-249098775.

13. Wang J, Zhou M, Liu F. Reasons for healthcare workers becoming infected with novel coronavirus disease 2019 (COVID-19) in China. J Hosp Infect [Internet]. 2020 [acesso em 06 abr 2020]. Disponível em: https://doi.org/10.1016/j.jhin.2020.03.002.

14. Jones S. Spain: doctors struggle to cope as 514 die from coronavirus in a day. The Guardian [Internet]. 2020 [acesso em 06 abr 2020]. Disponível em: https://www.theguardian.com/world/2020/mar/24/spaindoctors-lack-protection-coronavirus-covid-19.

15. Remuzzi A, Remuzzi G. COVID-19 and Italy: what next? The Lancet [Internet]. 2020 [acesso em 06 abr 2020]; 395. Disponível em: https://doi.org/10.1016/S0140-6736(20)30627-9.

16. Adams JG, Walls RM. Supporting the Health Care Workforce During the COVID-19 Global Epidemic. JAMA [Internet]. 2020 [acesso em 06 abr 2020]; 323(15). Disponível em: http://doi.org/doi:10.1001/ jama.2020.3972.

17. Mitchell G. Nurses among confirmed deaths from Covid-19 around the world. Nursing Times [Internet]. 2020 [acesso em 06 abr 2020]. Disponível em: https://www.nursingtimes.net/news/coronavirus/ nurses-among-confirmed-deaths-from-covid-19-around-the-world-20-03-2020/.

18. Conselho Federal de Enfermagem (COFEN). Covid-19 faz vítimas entre profissionais da saúde no Brasil. [Internet]. 2020 [acesso em 13 abr 2020] Disponível em: http://www.cofen.gov.br/covid-19-fazvitimas-entre-profissionais-da-saude-no-brasil 78979.html.

19. Agência Nacional de Vigilância Sanitária (BR). Gerência de Vigilância e Monitoramento em Serviços de Saúde. Gerência Geral de Tecnologia em Serviços de Saúde. Nota Técnica n. 04, de 21 de março de 2020. Orientações para serviços de saúde: medidas de prevenção e controle que devem ser adotadas durante a assistência aos casos suspeitos ou confirmados de infecção pelo novo coronavírus (sars-cov-2) [Internet]. Brasília: ANVISA; 2020 [acesso em 13 abr 2020]. Disponível em: https://www20.anvisa.gov. br/segurancadopaciente/index.php/alertas/item/nota-tecnica-n-04-2020-gvims-ggtes-anvisa-atualizadaem-21-03-2020.

20. Agência Nacional de Vigilância Sanitária (BR). Gerência de Vigilância e Monitoramento em Serviços de Saúde. Gerência Geral de Tecnologia em Serviços de Saúde. Nota Técnica n. 04, de 31 de março de 2020. Orientações para serviços de saúde: medidas de prevenção e controle que devem ser adotadas durante a assistência aos casos suspeitos ou confirmados de infecção pelo novo coronavírus (sarscov-2) [Internet]. Brasília: DF, 2020. [Acesso em: 13 abr 2020]. Disponível em: http://portal.anvisa.gov.br/ documents/33852/271858/Nota+T\%C3\%A9cnica+n+04-2020+GVIMS-GGTES-ANVISA/ab598660-3de44f14-8e6f-b9341c196b28.

21. Greenberg N, Docherty M, Gnanapragasam S, Wessely S. Managing mental health challenges faced by healthcare workers during covid-19 pandemic. BMJ [Internet]. 2020 [acesso em 13 abr 2020]; 368.

Disponível em: https://doi.org/10.1136/bmj.m1211.

22. Fisher $\mathrm{D}$, Wilder-Smith $\mathrm{A}$. The global community needs to swiftly ramp up the response to contain 
COVID-19. The Lancet [Internet]. 2020 [acesso em 13 abr 2020]; 395. Disponível em: https://doi. org/10.1016/S0140-6736(20)30679-6.

23. World Health Organization (WHO). What is World Health Day About? [Internet]. Geneva: WHO; 2020 [acesso em 13 abr 2020] Disponível em: https://www.who.int/news-room/campaigns/world-health-day/ world-health-day-2020.

24. International Council of Nurses. Nursing Now. [Internet] 2020 [acesso em 13 abr 2020]. Disponível em: https://www.icn.ch/what-we-do/campaigns/nursing-now.

Recebido: 07/04/2020

Finalizado: 05/05/2020

Editora associada: Luciana Puchalski Kalinke

Autor Correspondente:

Fernanda Moura D'Almeida Miranda

Universidade Federal do Paraná

Av. Lothário Meissner, 632 - 80210-170 - Curitiba, PR, Brasil

E-mail: fernandamiranda@ufpr.br

Contribuição dos autores:

Contribuições substanciais para a concepção ou desenho do estudo; ou a aquisição, análise ou interpretação de dados do estudo - FMAM, LLS, ACP, LMMS

Elaboração e revisão crítica do conteúdo intelectual do estudo - FMAM, LLS, ACP, LMMS

Aprovação da versão final do estudo a ser publicado - FMAM

Responsável por todos os aspectos do estudo, assegurando as questões de precisão ou integridade de qualquer parte do estudo - FMAM 